\title{
Multi-Objective Robust Design of New Rotate Barrel Based on Satisfaction Function
}

\author{
Hanzheng SUN, Zhengbao LEI, Yupeng HUANG, Jianghong Yu
}

\begin{abstract}
In this paper, a multi-objective robust design method based on satisfaction function was proposed by combining satisfaction function with Taguchi robust to solve the multi-objective optimization problem, which was easily interfered by noise factor. This method converted the signal-to-noise ratio of product quality characteristics into the expected smaller-the-better of Taguchi robust design, and realized the multi-objective robust design by weighted geometric mean, so as to solve the multi-objective optimization problem easily affected by noise factors. Under the premise of without changing rotate barrel of basic size by LS-DYNA FE model of rotary guardrail, the proposed method was carried out on the rotate barrel of multi-objective robust design, in order to solve the new rotary guardrail section parameter uncertain multi-objective optimization design. The results showed that the robust design of the new rotate barrel could resist the interference of the noise, the structure was more robust, and it conformed to the relevant laws and regulations by which was realized the purpose of lightweight of the new rotary guardrail. The research results had certain theoretical and engineering significance in improving the robustness of the new rotary guardrail.
\end{abstract}

Keywords: multi-objective; rotate barrel; satisfaction function; signal-to-noise ratio; Taguchi robust design

\section{INTRODUCTION}

Much of the current research in product design was driven by the fact that all real world engineering systems were comprised of uncertain parameters [1-3]. Therefore, the field of structural optimization needs to carry out more extensive research on design methods. For example, robust optimization which maximizes the robustness function, as well as the extreme case design [4-6]. Based on the orthogonal experimental design, Taguchi's robust design method played an important role in product design. In th early design stage, it could effectively reduce the sensitivity of product performance to noise fluctuas in $[7$, 8]. However, guardrail products were not a sing targo in traffic safety settings. Therefore, it was cessary to develop a new robust method for prod is th objective optimization design.

In practical engineering de, on, ulti-obje ve optimization problems were y ally trans rmed into single-objective problems by near weighting, uch as using genetic algorithms to o, imize proxy model [9]. Martin converted the ontimit 1 problen of multiobjective function co spor ing mul operating load into the optimizatio probler of sing ojective function by adopting the wo programming method [10]. McDonal a nd Heller incorporated robust designed strategies to du elop an iterative 2D FE optimization procedure, which was used to decide on precise shape of a hole in a plate to maximize its fatigue life [11]. Ozturk proposed an efficient method for fatigue based shape optimization to obtain the robust design of an oil sump shape with consideration of the variation of the clamping forces [12].

However the premise of these methods was that the research objects had not considered the influence of noise factors on products in real time. Therefore, in this paper the maximum acceleration of the sedan $(X, Y, Z)$ direction and the maximum lateral deviation of the guardrail after the passenger hit the guardrail were taken as the objective function. Through difference analysis and Variance analysis, the influence of noise factor was considered, a multi-objective robust design method put forward based on the satisfaction function, the satisfaction function method was introduced $i$ the tate $b$ rel of parameter optimization des of the pip ros ection. It helped to reduce the new e of ate bar structure noise factors on the sep avity andom actuations, improved the new tyr rotary a rdr of design parameters and objective fur tion robuness, achieved the goal of lic ight struo re.

\section{ROTARY 'GUARDRAIL ROBUSTNESS PROBLEM WITH MULTI-O JECTIVE SATISFACTION}

Optimization objectives such as guardrail products ver vultiple targets. In the optimization goal, structure usually needed to meet different design requirements at the same time, and set the traffic safety guardrail products as an example. It did not just want to control the vehicle collision guardrail in the three directions $(X, Y, Z)$, which must be less than the maximum acceleration ORA of $20 \mathrm{~g}$, but also required guardrail maximum offset $\Delta$ was less than $1000 \mathrm{~mm}$. Therefore, for the problem of multi-objective optimization design, the maximum deviation of guardrail should not exceed the threshold in the specification on the premise that the maximum acceleration of runaway vehicles was less than the standard value. Taken the rotate barrel as example, it was an important part of the rotary guardrail, when uncontrolled vehicle hit rotary guardrail by rotate barrel turning around columns, the vehicle drives out of the exercise direction of the return to normal, and it will rotate barrel between the above two guardrail beams.

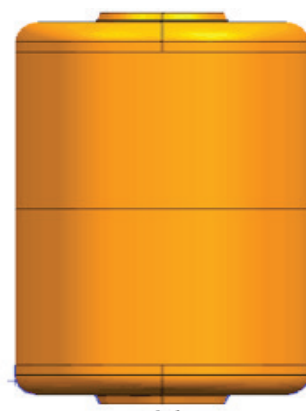

(a)

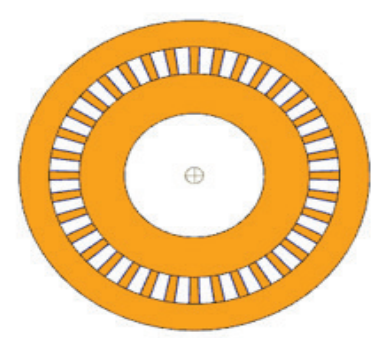

(b)
Figure 1 New rotate barrel: (a) New rotate barrel elevation; (b) New rotate barrel section 
Combined with the traditional rotary guardrail, the use of the process suffered from runaway vehicle affected, a new rotate barrel structure designed, and the basic configuration is shown in Fig. 1. The energy absorbing cavity structure in the outer circumference area of the upper part of rotate barrel was used to absorb the collision energy of runaway vehicles, and the middle part was used to drive the runaway vehicles to run in the normal track by rotating around the column, to achieve the purpose of using the guardrail.

\section{NEW ROTARY GUARDRAIL FE MODE ESTABLISHMENT AND VEHICLE FE MODEL VERIFICATION}

\subsection{FE Models of New Rotary Guardrail}

LS-DNYA was used to establish FE models of new rotary guardrail and vehicle, such as a new type of rotate barrel of the B-T differentiate four node shell element. The grid cell size was $15 \times 15 \mathrm{~mm}$, vehicle FE model was suitable for large deformation of the structure, which was used to 3 points of integral unit. The grid size of model units was controlled within $15 \mathrm{~mm}$, each grid unit warping angle of maximum angle control in less than $15^{\circ}$ and $135^{\circ}$, the triangular mesh number was controlled within 5\%. Fig. 2 shows the FE model of the new rotate barrel and the new rotary guardrail.

\subsection{Validation of Vehicle FE Modal for Topology Optimization}

The accuracy of the FE model of the vehicle was directly related to the reliability of the simulation results. Before the simulation test, this paper intended to comprehensively evaluate the correctness of the vehicle FE model by qualitative and quantitative aspects of the FE model of the $10 \mathrm{t}$ passenger and the 1,5 $\mathrm{t}$ sedan. As shown in Tab. 1, the geometric parameters tables of $10 \mathrm{t}$ passenger and 1,5 $\mathrm{t}$ sedan were given, the relevant parameters shown in Tab. 1, the FE models of $10 \mathrm{t}$ passenger and 1,5 sedan were established. When the uncontrolled vehicle collides with the guardrail, there is contact between them. In order to prevent the contact between the out-of-control vehicle and the guardrail, the guardrail and the uncontrolled vehicle model adopted Automatic-Single-Surface, the contact method that de self-contact between the guardrail and the ou control hicle. At the same time, the contact betwen th test vehic and the guardrail is adopted. By $d$ mition o Autom ic-Surface-to-Surface, the set dyne and atic a coefficients were 0,30

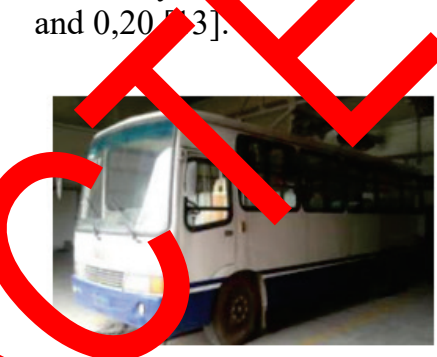

(a)

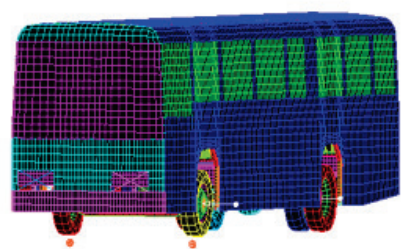

(b)
Pryare 3 Real passenger and FE model: (a) Real passenger; (b) FE passenger

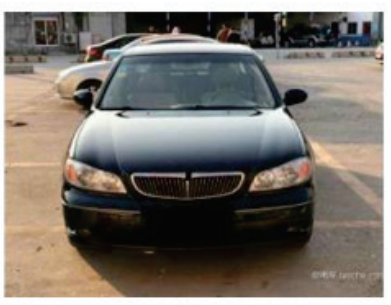

(a)

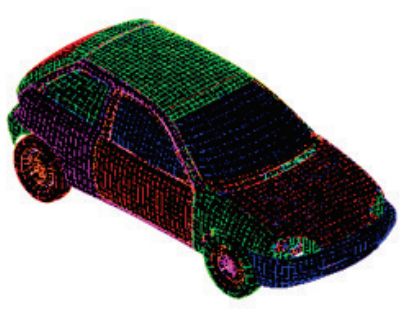

(b)
Figure 4 Real sedan and FE model: (a) Real sedan; (b) FE sedan

As shown in Fig. 3, Fig. 4, the real vehicle and FE vehicle of the $10 \mathrm{t}$ passenger and the 1,5 $\mathrm{t}$ sedan, and the collision test conditions described in Tab. 1 were set with reference to the relevant specifications $[14,15]$.

Table 1 The main parameter of vehicle mode

\begin{tabular}{|c|c|c|c|c|c|c|c|}
\hline \multirow{2}{*}{ Vehicle type } & \multirow{2}{*}{ Vehicle quality / $\mathrm{t}$} & \multicolumn{3}{|c|}{ Vehicle size / mm } & \multicolumn{3}{|c|}{ Vehicle centroid coordinates / mm } \\
\cline { 3 - 8 } & & Length & Width & Height & $X$ & $Y$ & $Z$ \\
\hline Passenger & 10 & 9020 & 2440 & 2880 & 4510 & 1220 & 1100 \\
\hline Sedan & 1,5 & 3750 & 1586 & 1433 & -1870 & 23 & 492 \\
\hline
\end{tabular}

Table 2 Material parameters of vehicle and guardrail

\begin{tabular}{|c|c|c|c|c|c|}
\hline Simulationmodal & Density $/ \mathrm{kg} / \mathrm{m}^{3}$ & Elasticity modulus / $\mathrm{MPa}$ & Poisson ratio / - & Yield strength / MPa & $\begin{array}{c}\text { Failure equivalent } \\
\text { Plastic strain }\end{array}$ \\
\hline Passenger & 7870 & $2,10 \times 10^{5}$ & 0,30 & 235 & 0,75 \\
\hline Sedan & 7870 & $2,10 \times 10^{5}$ & 0,30 & 235 & 0,75 \\
\hline Rotate barrel & 150 & $5,78 \times 10^{3}$ & 0,25 & 23.5 & 0,22 \\
\hline column & 7870 & $2,10 \times 10^{5}$ & 0,30 & 235 & 0,75 \\
\hline Profiled bar & 7870 & $2,10 \times 10^{5}$ & 0,30 & 235 & 0,75 \\
\hline U Block & 7870 & $2,10 \times 10^{5}$ & 0,30 & 235 & 0,75 \\
\hline
\end{tabular}




\subsubsection{Verification of Passenger FE Model}

Fig. 5, Fig. 6 were the trajectories of the real passenger and simulate passenger impact guardrail. It could be seen that during the collision process between the guardrail and the passenger under no abnormal driving conditions, the driving trajectory was the same.

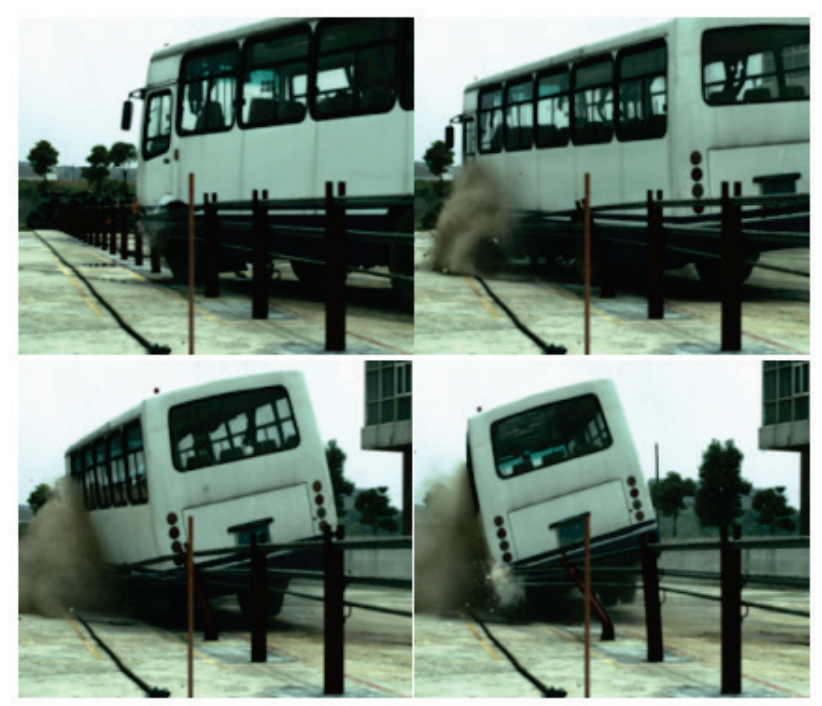

Figure 5 The motion condition of real passenger evaluation. Fig. 7 presents the acceleration curves in $(X, Y$, $Z$ ) at the mass center of the real passenger test, while their maximum values were $O R A_{x}=2,65 g, O R A_{Y}=1,94 g$, and $O R A_{z}=2,93 g$, respectively. The acceleration curves in the three directions at the mass center of the simulated test passenger are depicted in Fig. 8, and the maximum acceleration values were $O R A_{x}=2,75 g, O R A_{Y}=2,04 g$, and $O R A_{z}=3,05 g$. By comparing Fig. 7, Fig. 8, it was revealed that the maximum acceleration error in various directions did not exceed $5 \%$.

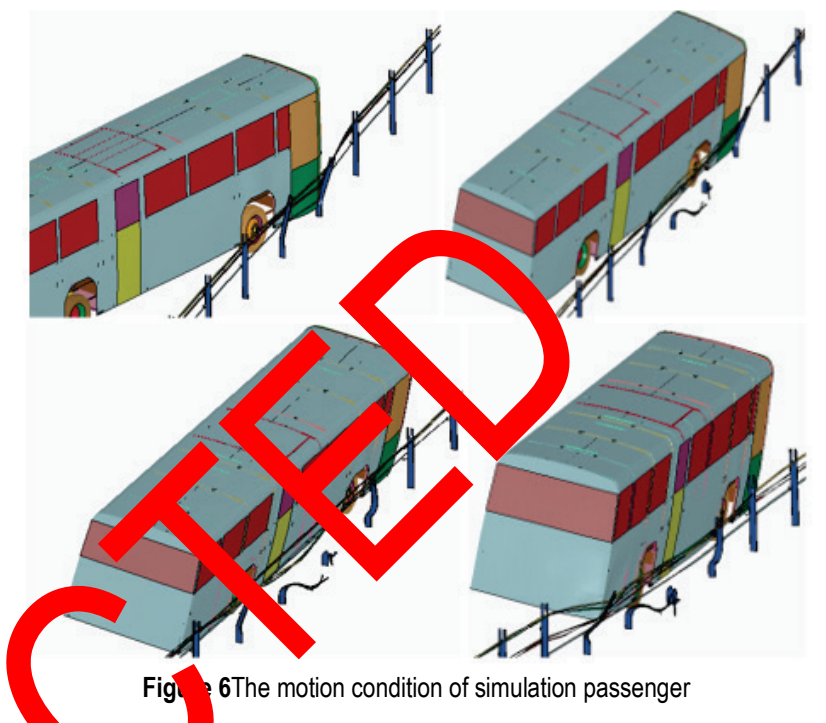

Herein, the maximum acceleration values at the center of mass of the vehicle were used for quantitative

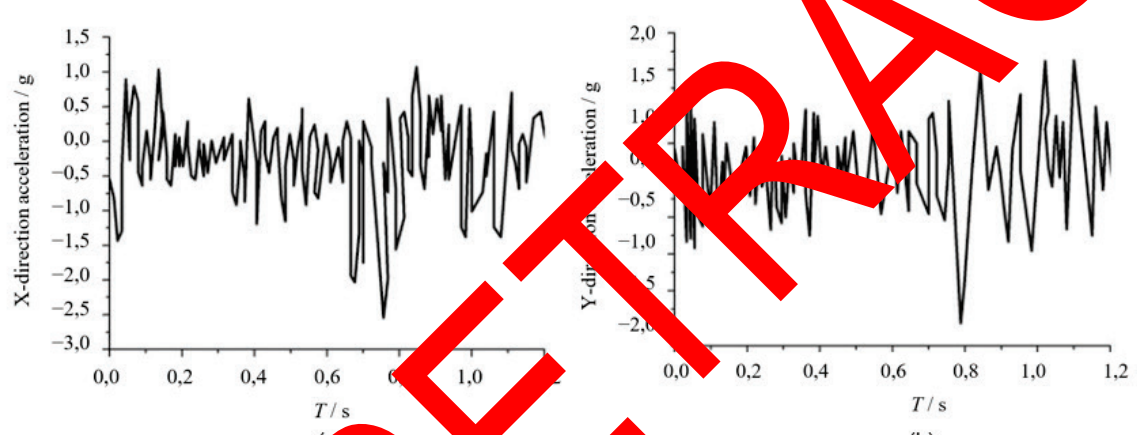

(b)

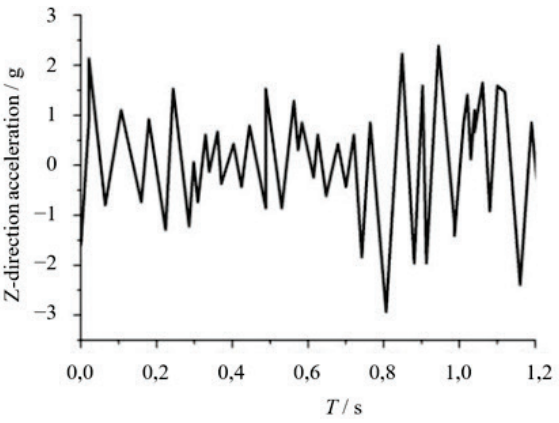

(c)

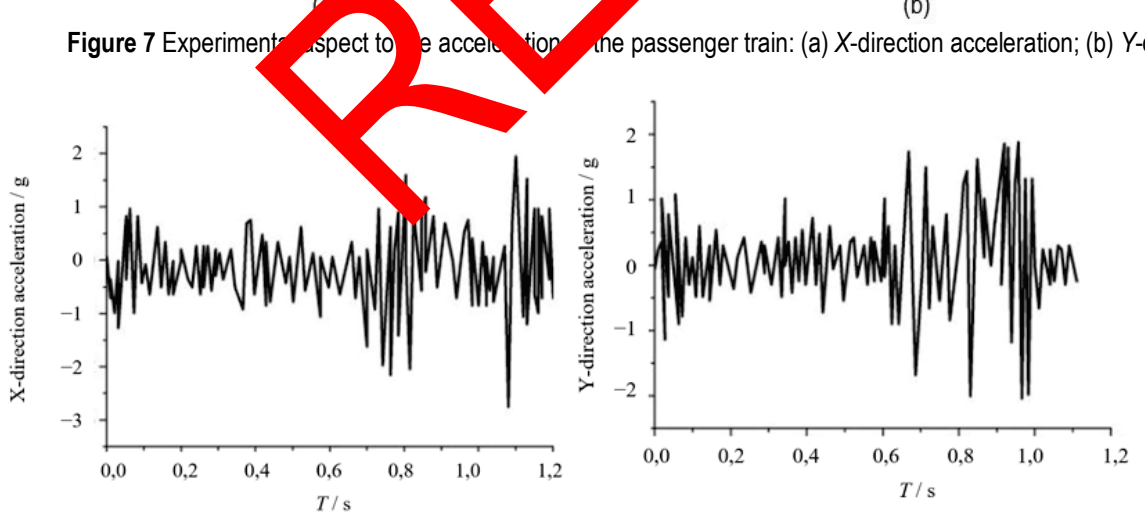

(a)

(b)

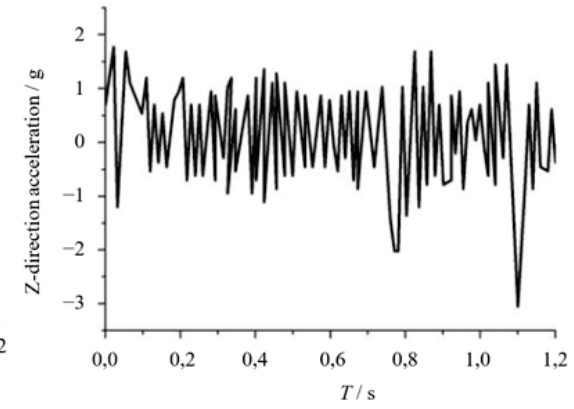

(c)

Figure 8 Simulation aspect to the acceleration of the passenger train: (a) X-direction acceleration; (b) Y-direction acceleration; (c) Z-direction acceleration

\subsubsection{Verification of Sedan Car FE Model}

Fig. 9, Fig. 10 were respectively the $1,5 \mathrm{t}$ sedan simulation test and the running track of the real vehicle test vehicle. It could be seen that during the collision between the vehicle and the guardrail, the vehicle did not show the phenomenon of drilling and smashing, and the collision trajectory of the vehicle and the guardrail was the same. As could be seen from Fig. 11, the damage positions of the actual and simulated were the same.

Fig. 12, Fig. 13 are divided into the maximum acceleration curves in the three directions of the vehicle's 
center of mass in the actual sedan and simulated sedan crash test. The actual vehicle mass acceleration values were: $O R A_{X}=12,67 g, O R A_{Y}=12,38 g, O R A_{Z}=11,41 g$. The acceleration value of the simulated vehicle centroid was: $O R A_{X}=13,25 g, O R A_{Y}=13,01 g, O R A_{Z}=12,02 g$. It could be seen that the error values of the maximum acceleration values in the three directions of the real vehicle and the simulated vehicle were all controlled within 5\%.
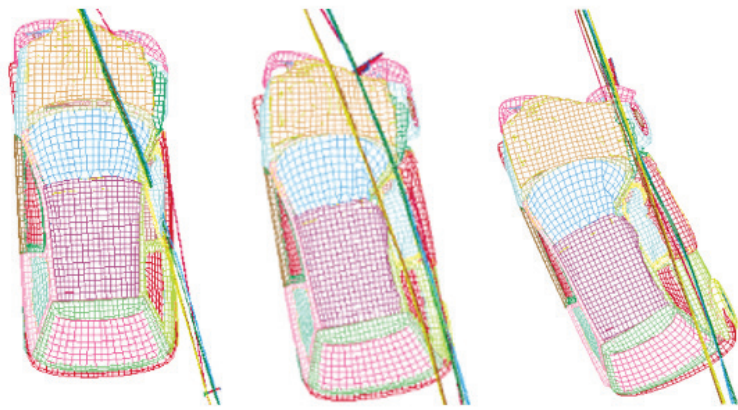

Figure 9 The motion condition of simulation sedan

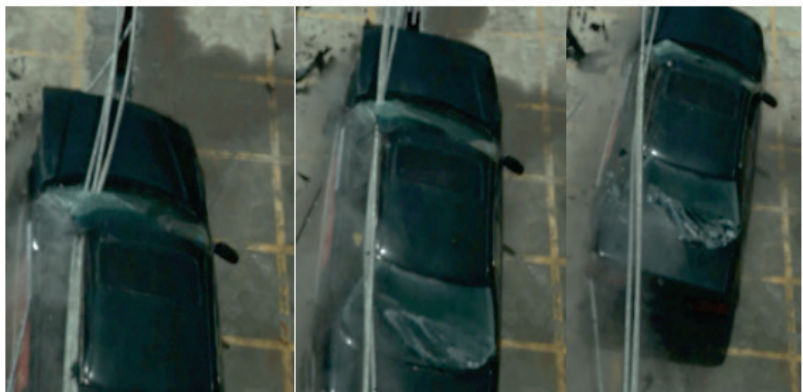

Figure 10 The motion condition of real sedan

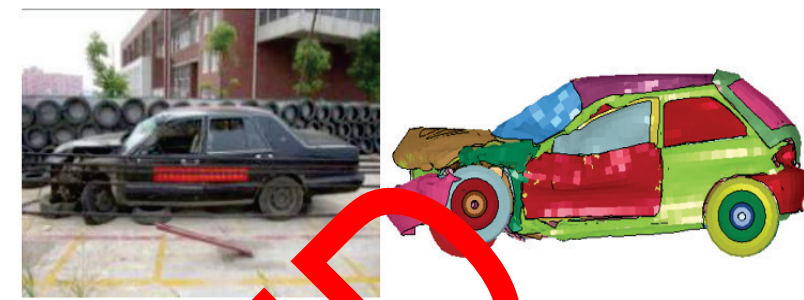

Figure 11 Damage nparison gram of re sedan and simulation sedan

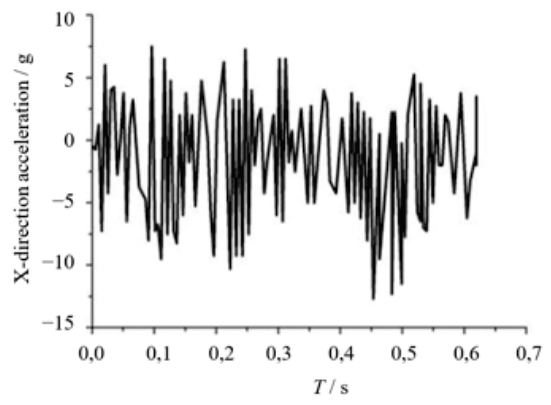

(a)

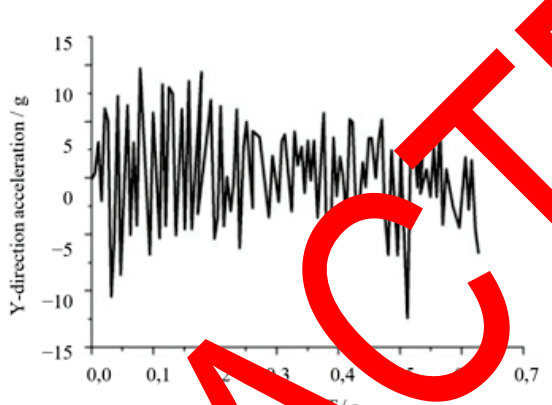

$>(b)$

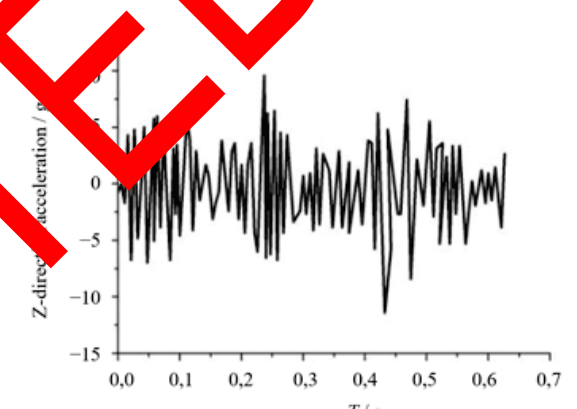

(c)

Figure 12 Experimental aspect to the acceleration of crea. dan: (a) direction acceleration; (b) Y-direction acceleration; (c) Z-direction acceleration

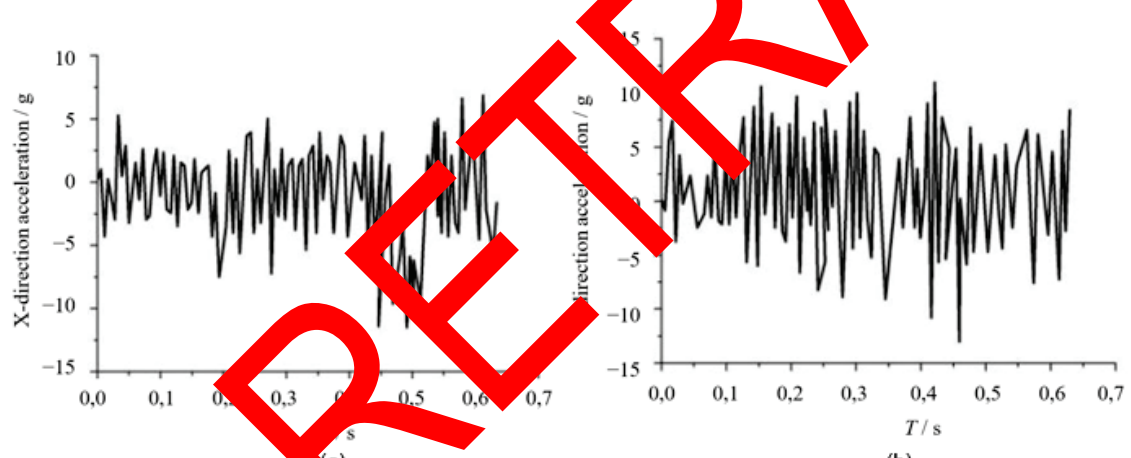

(b)

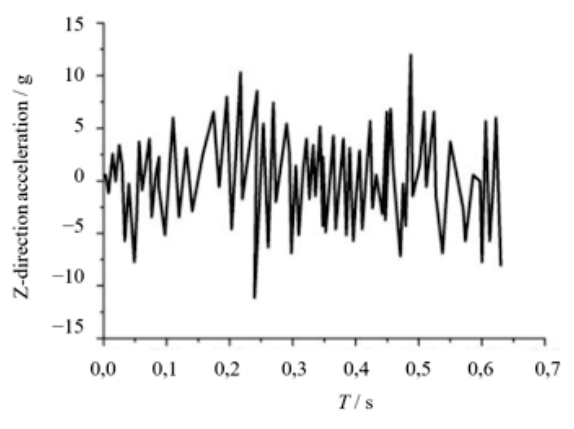

(c)

Figure 13 Simulatio spect to the acceleration of the sedan: (a) X-direction acceleration; (b) Y-direction acceleration: (c) Z-direction acceleration

Through the qualitative and quantitative analysis of the FE model of the 1,5 $\mathrm{t}$ sedan, the FE model of the 1,5 $\mathrm{t}$ sedan, the actual vehicle running state and damage degree were basically consistent with the real vehicle, and the acceleration error was within $5 \%$, so the 1,5 $\mathrm{t}$ sedan FE was reliable in future simulation work.

In the course of rotate barrel and guardrail rushing, it was used Cowper-Symonds constitutive model taking strain rate effect into account:

$\sigma_{\mathrm{Y}}=\left[1+\left(\frac{\varepsilon^{\prime}}{C}\right)^{\frac{1}{P}}\right]\left(\sigma_{0}+f_{n}\left(\varepsilon_{\mathrm{eff}}^{P}\right)\right)$

where $\sigma_{\mathrm{Y}}$ is the stress considering strain rate $\varepsilon^{\prime}, C$ and $P$ represent material strain rate parameter, $\sigma_{0}$ is single-axis tensile stress, $f_{n}\left(\varepsilon_{\text {eff }}^{p}\right)$ is the hardening function based on the effective plastic strain [16]. In this paper Eq. (1) $C$ is $40, P$ is 5 .

\section{SATISFACTION FUNCTION MULTI-OBJECTIVE ROBUSTNESS DESIGN METHOD}

The concept of satisfaction was to learn from the multiobjective decision making problem in statistics. After one thing appeared, it was judged whether satisfied or not based on people's subjective desires. As shown in Fig. 14, a satisfactory procedure was indicated by $q, q=1$ was fully satisfied, and $q=1$ was completely dissatisfied, where $q \in(0,1)$. 


$$
q_{i}=\frac{1}{1+\exp \left(2-4 \frac{C_{i}-C_{i, \max }}{C_{i l}-C_{i, \max }}\right)}
$$

In Eq. (2), $C_{i}$ represented the response value of each single target, $q_{i}$ represented the satisfaction value corresponding to the different response values of $C_{i}, C_{i, \max }$ was the lowest satisfaction value of the $i^{\text {th }}$ sub-target, and $C_{i l}$ was the ideal value of the satisfaction of the $i^{\text {th }}$ subtarget satisfaction.

\subsection{Signal-to-Noise Ratio}

Signal-to-noise ratio is an indicator used to measure fluctuations in product data. A larger signal-to-noise ratio means less quality loss, and the product is more robust. Signal-to-noise ratio (SNR) could be classified from its characteristics: nominal-the-better characteristic (NTB), smaller-the-better characteristic (STB), larger-the-better characteristic (LTB). Assumed that the measured values of the quality characteristics of the product were $y_{1}, y_{2}, \ldots, y_{n}$, and $T$ were the target values. Then the signal-to-noise ratio $S / N$ of the output response could be expressed as:

established to convert the signal-to-noise ratio of different product quality characteristics into satisfaction, while at the same time it is satisfying the STB in the robust design. Although the satisfaction function of STB was converted, its expression could be defined as Eq. (5):

$$
d_{i}(\eta)=\left\{\begin{array}{cc}
1 & \eta \leq \eta_{\min } \\
\left(\frac{\eta-\eta_{\min }}{\eta_{\max }-\eta_{\min }}\right) & \eta_{\min }<\eta<\eta_{\max } \\
0 & \eta_{\max } \leq \eta
\end{array}\right.
$$

Where: $d_{i}(\eta)$ was the satisfaction value of the output response function $Y_{i}$, and $0 \leq d_{i}(\eta) \leq 1,0 \leq r \leq 1, \eta_{\max }$, and $\eta_{\min }$ were the upper and lower limits of the signal-tonoise ratio compared with the output response value, and the values were calculated actu conditions and related data. When evaluating th satisfactio of multiple goals, it was necessary to conv multip single-objective satisfaction functi is into a 1 sati action function for comprehensive aluatio and th overall satisfaction $D(x) \in(0,1$ ouls a pressed as Eq. (6)

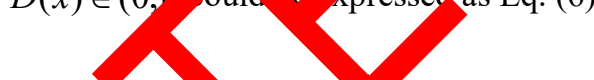

$D(x)=\left(d_{1} \times d_{2}, \times d_{k}\right) k$

$$
S / N= \begin{cases}-10 \lg \left(\frac{1}{n} \sum_{u=1}^{n} y_{u}^{2}\right) & \text { STB } \\ -10 \lg \left(\frac{1}{n} \sum_{u=1}^{n}\left(\frac{1}{y_{u}}\right)^{2}\right) & \text { LTB } \\ -10 \lg \left(\frac{1}{n} \sum_{u=1}^{n}\left(y_{n}-T\right)^{2}\right) & \text { NTB }\end{cases}
$$

here: $d_{k} \mathrm{~W}$ the satisfaction of the $j^{\text {th }}$ sub-reaction, where $j, 1,2, \ldots$, ,

2 Optimization Design of Section Parameters of Rotate rel of New Rotary Guardrail

According to Standard for Safety performance Evaluation of Highway Barrier JTG B05-01-2013 [14], the safety performance of the guardrail should have the function of blocking, buffering and guiding. It was forbidden for any vehicle to cross, climb or ride guardrail in any form. In this paper, the maximum acceleration $O R A$ in the $(X, Y, Z)$ directions of the sedan's center of mass and the maximum lateral offset $\Delta$ of the guardrail after the collision of the passenger were used as the objective function. Therefore, in order to unify the evaluation index, the maximum acceleration ORA and the maximum lateral offset $\Delta$ could be dimensionlessly processed. (This paper stipulated the maximum acceleration $O R A \geq 16 \mathrm{~g}$ and the maximum lateral displacement $\Delta \geq 800 \mathrm{~mm}$, and the satisfaction value of the output response was 0 , and the overall conversion was the overall satisfaction as the evaluation index).

\subsubsection{Determination of Orthogonal Test Factors and Levels}

Under the premise of ensuring, the inner diameter is $r_{1}$ $=150 \mathrm{~mm}$, and outer diameter is $r_{2}=150 \mathrm{~mm}$ of the new rotate barrel. The design factors for the cross-section configuration of the new rotate barrel were determined initially-the outer wall thickness $A$, the outer support plate thickness $B$, the support plate length $C$, and the inner support plate thickness $D$, which were the experimental factors for the orthogonal test. A new rotate barrel section configuration was shown in Fig. 14. 


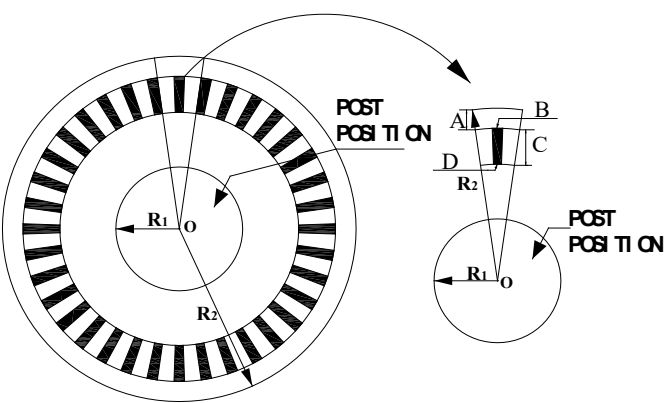

Figure 14 New section configuration of Rotate Barrel

In the orthogonal test analysis of the new rotate barrel section, each factor was selected for 4 factors and 4 levels, and the horizontal range of each factor was: $22 \leq A \leq 31$; $4,0 \leq B \leq 7,0 ; 25 \leq C \leq 34 ; 4,0 \leq D \leq 7,0$.

Table 3 Factors and level of new type of rotate barrel

\begin{tabular}{|c|c|c|c|c|}
\hline \multirow{2}{*}{ LEVEL } & \multicolumn{4}{|c|}{ FACTOR / mm } \\
\cline { 2 - 5 } & $A$ & $B$ & $C$ & $D$ \\
\hline 1 & 22 & 4,0 & 25 & 4,0 \\
\hline 2 & 25 & 5,0 & 28 & 5,0 \\
\hline 3 & 28 & 6,0 & 31 & 6,0 \\
\hline 4 & 31 & 7,0 & 34 & 7,0 \\
\hline
\end{tabular}

The orthogonal test was performed by using $L_{16}\left(4^{4}\right)$ orthogonal table, and the orthogonal test was generated by SPSS (statistical product and service solutions). The collision conditions were as shown in Tab. 4, and the results of related data collection are shown in Tab. 5 .

Extremum difference analysis method was used to analyse the test data in Tab. 5. The range was also c. the variation amplitude, which reflected the maxim $n$ degree of dispersion in a group of data. The larger th difference, the greater the influence of the actor $n$ the result. Eq. (7) was shown.

$$
R=\max \left\{\bar{K}_{i}\right\}-\min \left\{\bar{K}_{i}\right\}
$$

where: $K_{i}$ was the average of the deviations of the $i^{\text {th }}$ level.

Table 4 Rotary guardrail collision working table

\begin{tabular}{|c|c|c|c|}
\hline Vehicle model & $\begin{array}{c}\text { Collision velocity } \\
\text { / km/h }\end{array}$ & $\begin{array}{c}\text { Collision } \\
\text { angle/ }{ }^{\circ}\end{array}$ & $\begin{array}{c}\text { Vehicle mass / } \\
\mathrm{t}\end{array}$ \\
\hline Sedan car & 100 & $20^{\circ}$ & 1,5 \\
\hline Passenger car & 60 & $20^{\circ}$ & 10 \\
\hline
\end{tabular}

According to extremum difference analysis in Eq. (7), the average response values of the overall satisfaction of the maximum acceleration $O R A$ and the maximum transverse offset $\Delta$ corresponding to the design factors of the new rotate barrel section were shown in Fig. 15 The overall satisfaction was larger and more satisfactory from Fig. 16, the optimal combination of the orthogonal test of new rotate barrel section $, B_{2}, C_{1}, D_{3}$. That was, the outer wall thickness as $25 \mathrm{~m}$ the outer support plate thickness was $5 \mathrm{~mm}$, support $\mathrm{A}$ te length was $25 \mathrm{~mm}$, and the inner sy ort pla thickne was $6 \mathrm{~mm}$.

Tab Statistical ta of orthogonal test results of new rotary guardrail

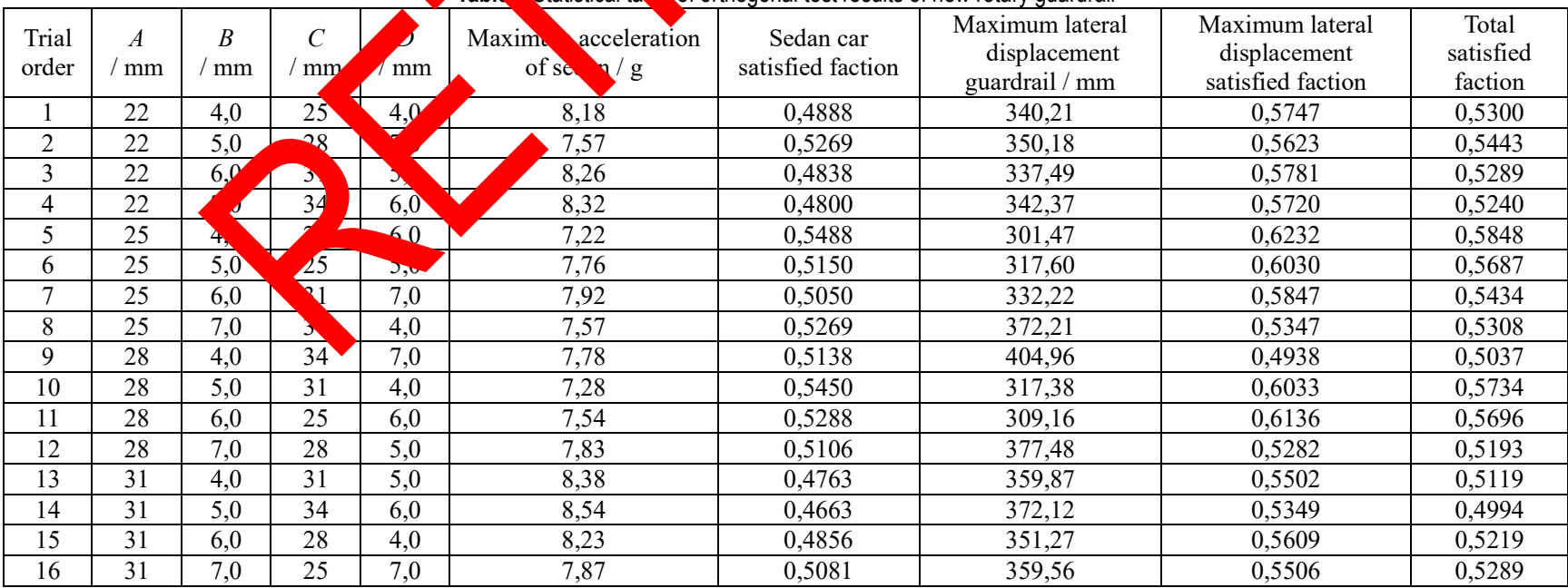

Because of the range analysis, the variance analysis could be used to determine the contribution rate of the overall satisfaction of the new rotate barrel section design factors as shown in Fig. 16. The design factors were based on the contribution rate of the corresponding overall factors $A, C, B, D$. 


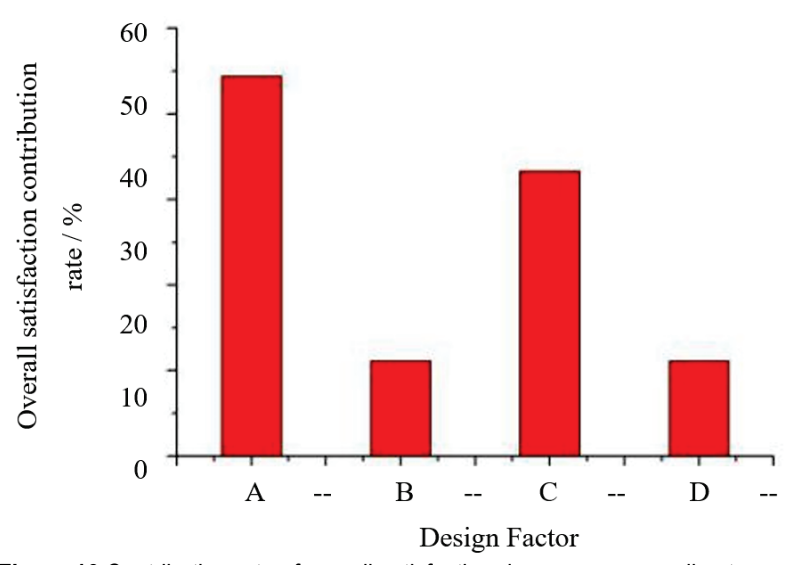

Figure 16 Contribution rate of overall satisfaction degree corresponding to each design factor of the new rotate barrel section

\subsection{Multi-Target Robust Design of New Rotary Guardrail 4.4.1 Controllable Factors and Noise Factor Selection}

The traditional parameter optimization design did not consider the influence of the noise factor on the test results, but the noise factor exists in real time. In order to improve the robustness of the new rotate barrel structure, according to the variance analysis shown in Fig. 17, the two factors $A$ and $C$ were the controllable factors of the robust design, as shown in Tab. 6.

According to the vehicle quality tolerance error, the collision speed tolerance error, and the collision angle tolerance error specified in the literature $[15,16]$, the multiobjective robustness design was performed as shown Tab. 7.

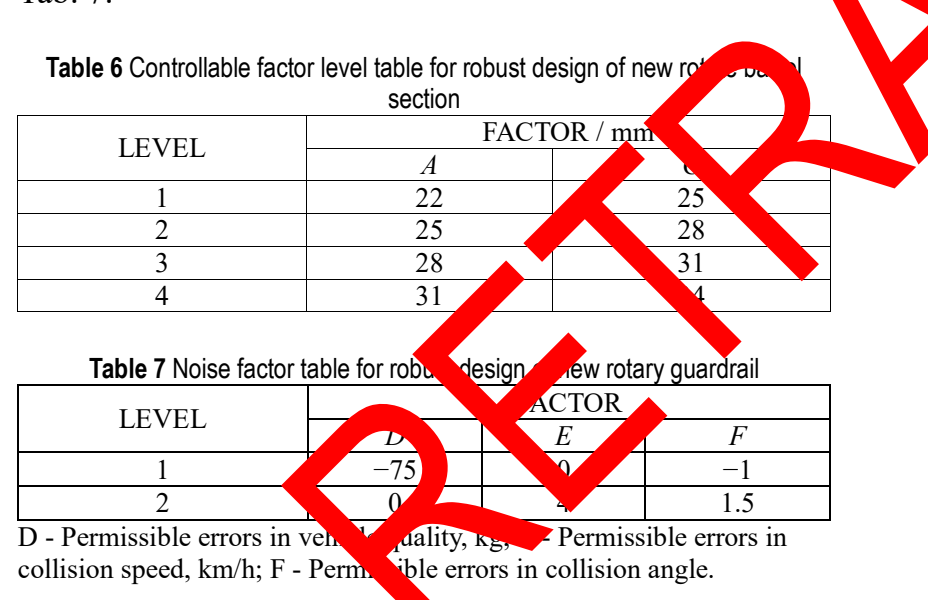

\subsubsection{Optimal Combination Anarysis of Multi-Objective Robust Design}

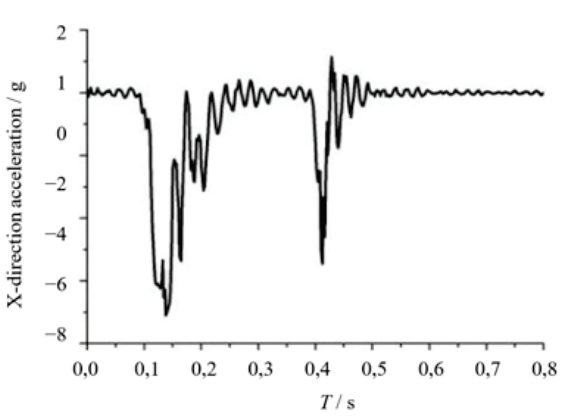

(a)

The mean maximum acceleration, the mean maximum offset of guardrail, and Overall satisfaction of the new rotate barrel obtained after multi-objective robustness optimization design were shown in Tab. 8.

By extremum difference analysis method as shown in Fig. 18, the optimal combination of controllable factors in the design of robustness was: $A_{3}, C_{3}$, the outer wall thickness was $28 \mathrm{~mm}$, the support plate length was $31 \mathrm{~mm}$.

By variance analysis, the corresponding contribution rate of the overall satisfaction corresponding to each controllable factor was shown in Fig. 18. The contribution rate was $C$ and $A$ by the orthogonal test. The final determination of the optimal combination of robustness was the outer wall thickness $28 \mathrm{~mm}$, the outer support plate thickness $5 \mathrm{~mm}$, the support plate length $31 \mathrm{~mm}$ and the inner support plate thickness $6 \mathrm{~mm}$.

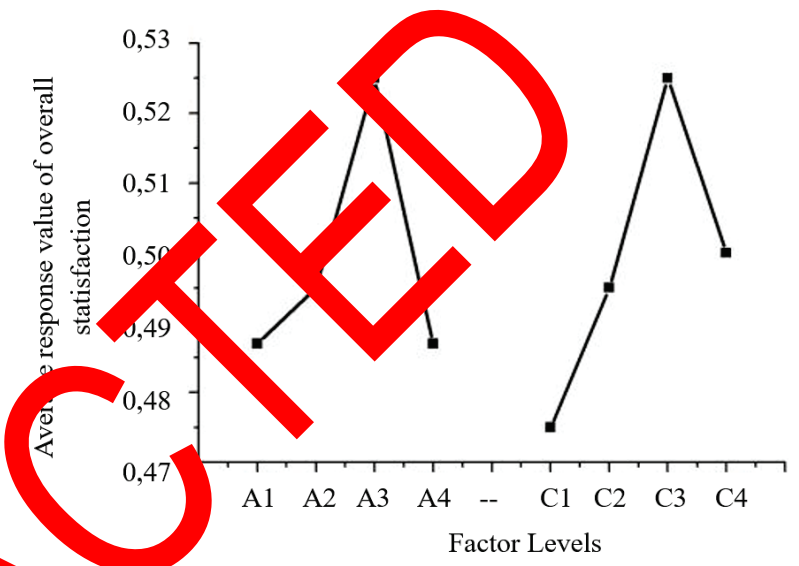

ure 17 Average response value of total satisfaction of controllable factors in robust design of new rotary guardrail

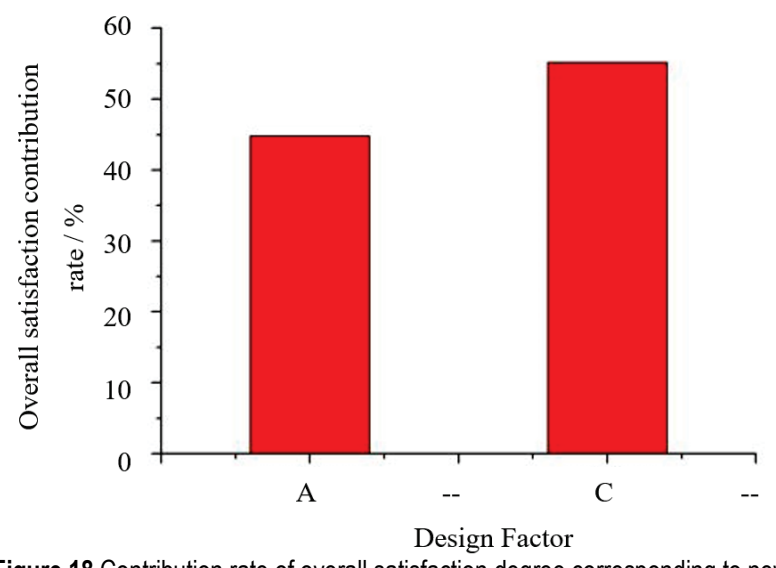

Figure 18 Contribution rate of overall satisfaction degree corresponding to new rotary guardrail

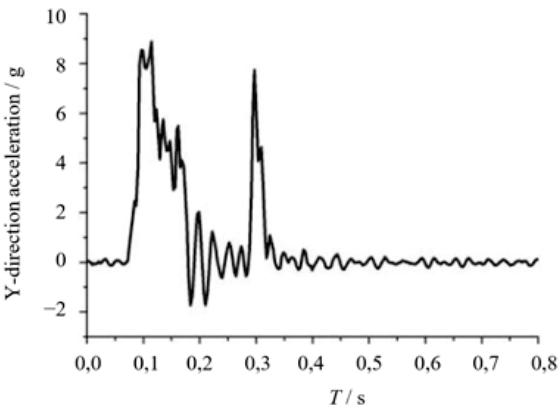

(b)

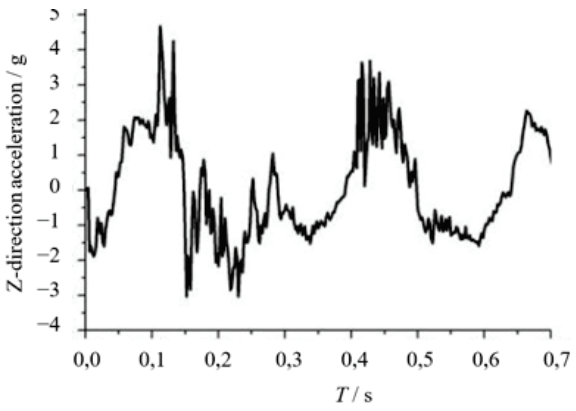

(c)

Figure 19 Optimization of the sedan before the center of mass acceleration: (a) X-direction acceleration; (b) Y-direction acceleration; (c) Z-direction acceleration 
Table 8 Average acceleration, maximum deviation of guardrail and overall satisfaction response value

\begin{tabular}{|c|c|c|c|c|c|c|c|}
\hline \multirow{2}{*}{ Trail order } & \multicolumn{3}{|c|}{ Mean maximum acceleration / $\mathrm{g}$} & \multicolumn{3}{|c|}{ Mean maximum offset of guardrail $/ \mathrm{mm}$} & \multirow{2}{*}{$\begin{array}{c}\text { Overall } \\
\text { satisfaction }\end{array}$} \\
\hline & $a$ & Sn2 & D1 (sn1) & $\triangle x$ & Sn2 & $\mathrm{D} 2(\mathrm{sn} 2)$ & \\
\hline 01 & 7,23 & $-17,18$ & 0,81 & 361,26 & $-51,16$ & 0,29 & 0,48 \\
\hline 02 & 7,54 & $-17,55$ & 0,77 & 347,68 & $-50,82$ & 0,30 & 0,48 \\
\hline 03 & 7,35 & $-17,33$ & 0,79 & 324,64 & $-50,23$ & 0,33 & 0,51 \\
\hline 04 & 7,13 & $-17,06$ & 0,83 & 372,19 & $-51,42$ & 0,28 & 0,48 \\
\hline 05 & 7,04 & $-16,95$ & 0,84 & 383,16 & $-51,67$ & 0,27 & 0,47 \\
\hline 06 & 7,08 & $-17,00$ & 0,83 & 340,86 & $-50,65$ & 0,31 & 0,51 \\
\hline 07 & 7,15 & $-17,09$ & 0,82 & 349,40 & $-50,87$ & 0,30 & 0,49 \\
\hline 08 & 7,04 & $-16,95$ & 0,84 & 351,27 & $-50,91$ & 0,30 & 0,50 \\
\hline 09 & 7,64 & $-17,67$ & 0,75 & 323,45 & $-50,20$ & 0,33 & 0,50 \\
\hline 10 & 7,11 & $-17,03$ & 0,83 & 334,61 & $-50,49$ & 0,31 & 0,51 \\
\hline 11 & 7,08 & $-17,00$ & 0,83 & 292,90 & $-49,34$ & 0,36 & 0,55 \\
\hline 12 & 7,06 & $-16,97$ & 0,84 & 333,15 & $-50,45$ & 0,32 & 0,51 \\
\hline 13 & 7,26 & $-17,22$ & 0,81 & 403,86 & $-52,13$ & 0,25 & 0,45 \\
\hline 14 & 7,12 & $-17,05$ & 0,83 & 384,89 & $-51,71$ & 0,26 & 0,47 \\
\hline 15 & 7,49 & $-17,49$ & 0,77 & 295,53 & $-49,41$ & 0,37 & 0,53 \\
\hline 16 & 6,97 & $-16,86$ & 0,85 & 367,80 & $-51,31$ & 0,28 & 0,49 \\
\hline
\end{tabular}

\section{DISCUSSION}

The traditional robust design method belonged to the single-objective optimization problem, which was difficult to achieve the multi-objective optimization and did not consider the impact of noise factors on products in real time. However, noise factors existed in actual product design. This method could solve the optimization problem of multi-objective robust design with noise factor. On the premise of keeping the basic size of rotate barrel section unchanged, the relevant parameters were obtained by adopting the robustness design of multi-objective satisfaction function considering the noise factor. The final determination of the optimal combination of robust was: the outer wall thickness $28 \mathrm{~mm}$, the outer supp plate thickness $5 \mathrm{~mm}$, the support plate lengt the inner support plate thickness $6 \mathrm{~mm}$. T parameters of rotate arro obtained by orthogonal experiment without se, the ou wall thickness was 25 $\mathrm{mm}$, and the oute supp plate th kness was $5 \mathrm{~mm}$. The support plate le sth was $2 . \mathrm{nm}$, an the inner support plate thickness w $6 \mathrm{~mm}$ y us celeration $O R A$ and its maximur ofts a double evaluation index, there is a declin in the nowab acceleration, and guardrail ma nus offset. L optimization the acceleration value at tho enter or mass of sedan was $O R A_{x}=7,5 \mathrm{~g}$, $\mathrm{OKA}_{Y}=8,88 \mathrm{~g}, Q R A_{Z}=5,24 \mathrm{~g}$ as shown in Fig. 19. The passenger maximum deviation of guardrail was 398,46 $\mathrm{mm}$. The ptimized acceleration value at the center of mass f sedan as $O R A_{x}=7,01 \mathrm{~g}, O R A_{Y}=6,73 \mathrm{~g}, O R A_{Z}=4,51 \mathrm{~g}$ as $\quad$ in Fig. 20, and the passenger maximum deviation fouardrail was $345,98 \mathrm{~mm}$. The maximum offset declined $52,48 \mathrm{~mm}$.

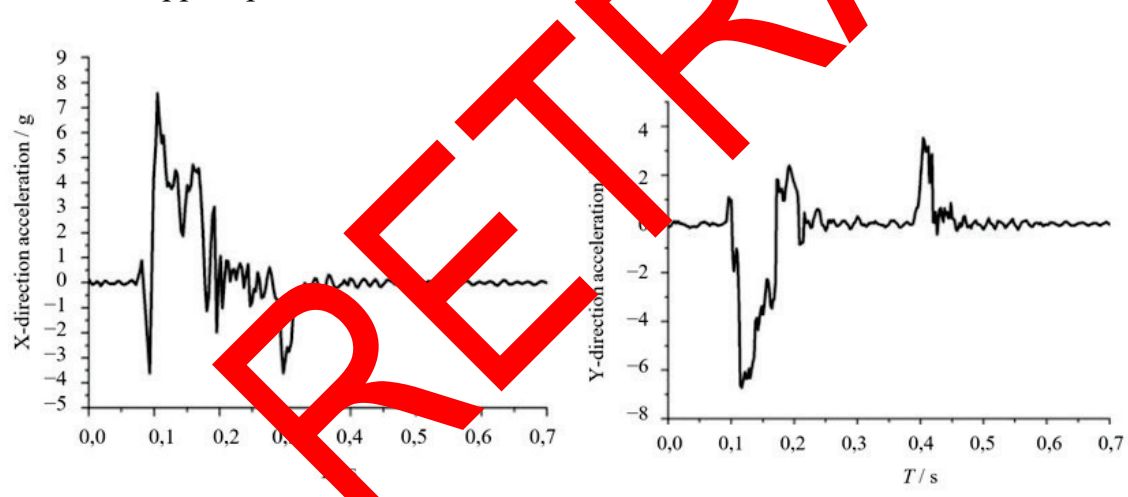

(a)

(b)

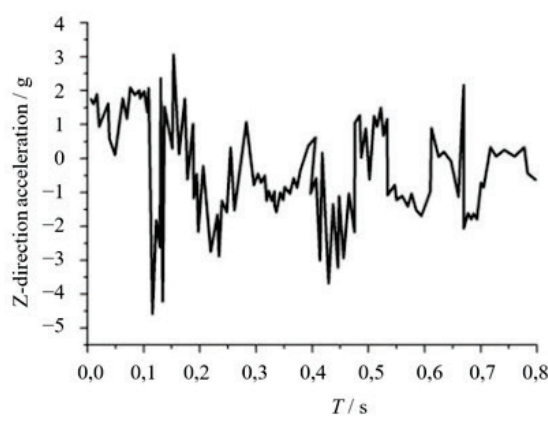

(c)

Figure 20 Optimization of the sedan after the center of mass acceleration: (a) X-direction acceleration; (b) Y-direction acceleration; (c) Z-direction acceleration

\section{CONCLUSIONS}

Aimed at the multi-objective optimization and the influence of noise problem, this paper combined the signalto-noise ratio and satisfaction function to establish a multiobjective robust design method based on satisfaction function, and utilized the multi-objective robust design method. The satisfaction function of the multi-objective robust design of the new rotating cylinder was studied. The robust design of the new rotating cylinder after the response mean and response standard deviation was reduced, and the collision FE analysis was set according to the relevant specifications. In the collision analysis of the new rotating guardrail with and without noise factors, the maximum acceleration and maximum deviation of the guardrail were reduced by 2,15 $\mathrm{g}$ and $52,48 \mathrm{~mm}$, achieving the goal of light weighting.

\section{Acknowledgments}

The research was supported by the National Natural Science Foundation of China (No. 51675059), Key Research and Development Project of Science and Technology Plan of Hunan Province (No. 2019SK2174), Hunan Provincial Innovation Foundation for Postgraduate (No. CX20190641). The authors also would like to thank 
the Large Structural Crash Testing Laboratory for its support.

\section{REFERENCES}

[1] Lee, S. H. \& Chen, W. (2009).A Comparative Study of Uncertainty Propagation Methods for Blacks-Box-Type Problems. Struct Multidiscip Optim, 37(3), 239-253. https://doi.org/10.1007/s00158-008-0234-7

[2] Schueller, G. I. \& Jensen, H. A. (2008).Computational Methods in Optimization Considering Uncertainties-An Overview. Comput Methods Appl Mech Engrg, 198(1), 2-13. https://doi.org/10.1016/j.cma.2008.05.004

[3] Moller, B. \& Beer, M. (2008). Engineering Computation Under Uncertainly-Capabilities of Non-Traditional Models. Comput Struct, 86(10), 1024-1041. https://doi.org/10.1016/j.compstruc.2007.05.041

[4] Hashimoto, D. \& Kanno, Y. (2015). A semidefinite programming approach to robust truss topology optimization under uncertainly in locations of nodes. Struct Multidiscip Optim, 51, 439-461. https://doi.org/10.1007/s00158-014-1146-3

[5] Ohsaki, M. \& Katsura, M. (2010). A random sampling approach to worst-case design of structures. Struct Multidisc Optim, 46(1), 27-39. https://doi.org/10.1007/s00158-011-0752-6

[6] Takezawa, A., Nii, S., Kitamura, M., \& Kogiso, N. (2011). Topology optimization for worst load conditions based on the eigenvalue analysis of an aggregated linear system. Comp Meth Appl Mech Engrg, 200(25-28), 2268-2281. https://doi.org/10.1016/j.cma.2011.03.008

[7] Abhiram, D. R. \& Gangulir, H. D. (2018). Robust design of small unmanned helicopter for hover performance using Taguchi method. Journal of Aircraft, 55(4), 1746-1753. https://doi.org/10.2514/1.C034539

[8] Lee, S, Kim, K, Cho, S, et al. (2014). Optimal design or interior permanent magnet synchronous motor considering the manufacturing tolerances using Taguchi rob IET Electric Power Applications, 8(1), 23-28. https://doi.org/10.1049/iet-epa.2013.0109

[9] Sekulski, Z. (2010). Multi-objective optimization of high-speed vehicle- enger ca naran structure by genetic algorithm. Ma ne uctures, - 4), 405-433. https://doi.org/10.1016/j.m struc.2010. 001

[10] Martin, M. A., Cuadrado, N L., \& Romero, (2011) Computing efficient fina al stra sies: An xtended compromise programming ap oa Applied Mathematics and Computation, 217837. https://doi.org/10.101 ,amc.2 1.02 .

[11] McDonald, M. Hell M. (2 4). Robust shape optimization of no cor rand life extension. Struct Multidiscip Optim, 28, -68. https://doi.org/10.1007/s001s 004-0437-5

[12] Ozturk, U. E. (2011). Efficrent method for fatigue based optimization of the oil sump carrying a differential case in four wheel drive vehicles. Struct Multidiscip Optim, 44, 823830. https://doi.org/10.1007/s00158-011-0678-z

[13] Yin, H., Xiao, Y., \& Wen, G. (2016). Multi-objective robust optimization of foam-filled bionic thin-walled structures. Thin-Walled Structures, 109, 332-343. https://doi.org/10.1016/j.tws.2016.10.011

[14] JTG B05-01-2013: Standard for Safety performance Evaluation of Highway Barrier. China: Ministry of Transport of the People's Republic of China.

[15] DB 33/T 888-2013: Specification for layout of rotary guardrail. China: Quality and Technology Supervision of Zhejiang Province.

[16] Peng, Y., Deng, W., \& Xu, P. (2015). Study on the collision performance of a composite energy-absorbing structures for subway vehicle. Thin-Walled Structures, 94, 663-672. https://doi.org/10.1016/j.tws.2015.05.016
Contact information:

\section{Hanzheng SUN}

Large Structural Crash Testing Laboratory,

Changsha University of Science \& Technology,

Changsha 410114, China

E-mail: 836233052@qq.com

\section{Zhengbao LEI}

(Corresponding author) Large Structural Crash Testing Laboratory,

Changsha University of Science \& Technology,

Changsha 410114, China

E-mail: doclei@163.com

\section{Yupeng HUANG}

Large Structural Crash Testing Laboratory,

Changsha University of Science \& Technology,

Changsha 410114, China

\section{Jianghong YU}

Large Structural Crash Testing Labor

Changsha University of Science \&

Changsha 410114, China

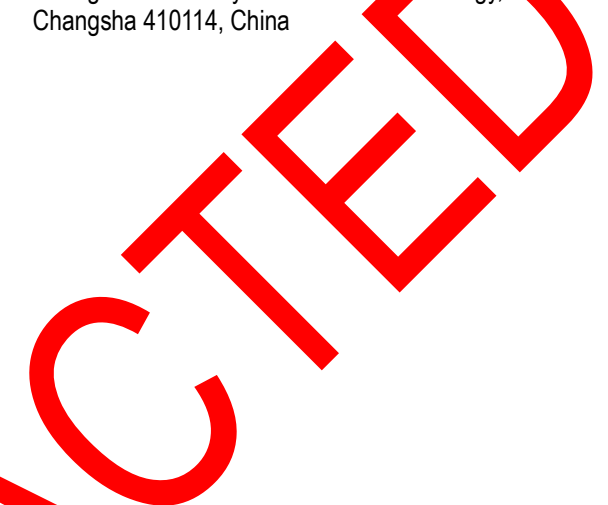

